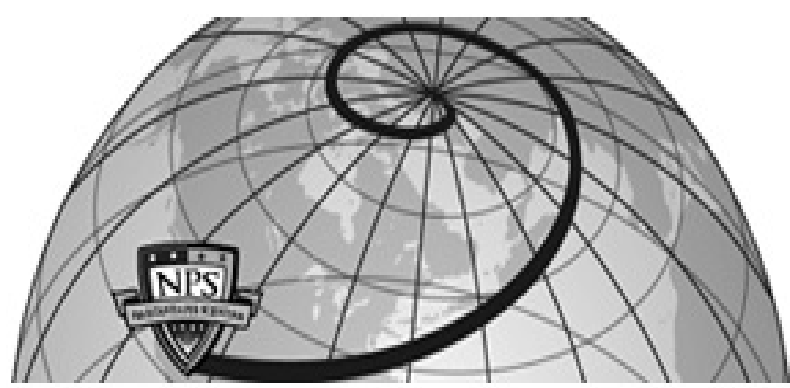

Calhoun: The NPS Institutional Archive DSpace Repository

Nodal high-order discontinuous Galerkin methods for the spherical shallow water equations

Giraldo, F.X.; Hesthaven, J. S.; Warburton, T.

Journal of Computational Physics / Volume 181, Issue 2, 499-525

https://hdl.handle.net/10945/25524

This publication is a work of the U.S. Government as defined in Title 17, United States Code, Section 101. Copyright protection is not available for this work in the United States.

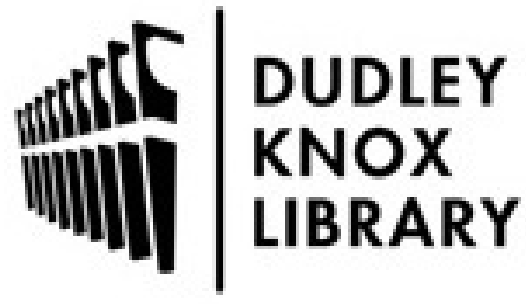

http://www.nps.edu/library
Calhoun is the Naval Postgraduate School's public access digital repository for research materials and institutional publications created by the NPS community. Calhoun is named for Professor of Mathematics Guy K. Calhoun, NPS's first appointed -- and published -- scholarly author.

Dudley Knox Library / Naval Postgraduate School 411 Dyer Road / 1 University Circle Monterey, California USA 93943 


\title{
Nodal High-Order Discontinuous Galerkin Methods for the Spherical Shallow Water Equations
}

\author{
F. X. Giraldo, ${ }^{*, 1}$ J. S. Hesthaven, $†$ and T. Warburton $\ddagger$ \\ * Naval Research Laboratory, Monterey, California 93943; $\dagger$ Division of Applied Mathematics, \\ Brown University, Providence, Rhode Island 02912; and $\ddagger$ Deptartment of Mathematics \\ and Statistics, University of New Mexico, Albuquerque, New Mexico 87131 \\ E-mail: giraldo@nrlmry.navy.mil
}

Received September 29, 2001; revised May 23, 2002

\begin{abstract}
We present a high-order discontinuous Galerkin method for the solution of the shallow water equations on the sphere. To overcome well-known problems with polar singularities, we consider the shallow water equations in Cartesian coordinates, augmented with a Lagrange multiplier to ensure that fluid particles are constrained to the spherical surface. The global solutions are represented by a collection of curvilinear quadrilaterals from an icosahedral grid. On each of these elements the local solutions are assumed to be well approximated by a high-order nodal Lagrange polynomial, constructed from a tensor-product of the Legendre-Gauss-Lobatto points, which also supplies a high-order quadrature. The shallow water equations are satisfied in a local discontinuous element fashion with solution continuity being enforced weakly. The numerical experiments, involving a comparison of weak and strong conservation forms and the impact of over-integration and filtering, confirm the expected high-order accuracy and the potential for using such highly parallel formulations in numerical weather prediction. (c) 2002 Elsevier Science (USA)

Key Words: discontinuous Galerkin method; filters; high-order; icosahedral grid; shallow water equations; spectral element method; spherical geometry.
\end{abstract}

\section{INTRODUCTION}

The majority of current climate and numerical weather prediction (NWP) models, e.g., the operational NWP models developed by the European Center for Medium-Range Weather Forecasting (ECMWF), the National Center for Environmental Prediction (NCEP), and the Naval Research Laboratory (NRL), are based on globally defined spectral methods

\footnotetext{
${ }^{1}$ Corresponding author.
} 
$[13,16,26,27]$. While these methods continue to be essential tools for NWP, their limitations are beginning to emerge. For one, the fixed global grid makes adaptive solution techniques very complex if possible at all. Furthermore, the recent paradigm shift in large-scale computing from vector to distributed-memory computing platforms has exposed problems with achieving efficiency due to the global, inter-processor, all-to-all communication needed in the spectral transform.

Such considerations have stimulated research into parallel methods and potentially adaptive methods, suitable for NWP, which maintain the accuracy of the global spectral approach while overcoming its inherent limitations.

Continuous spectral element methods (SEM) have recently been proposed for future climate [28] and NWP [10] models. In these methods the solution is first constructed in a local element-by-element manner and then a portion of this local solution is distributed to the global grid points shared by adjacent elements. This global assembly from the elements to the global grid points is what enforces continuity and it is this requirement which reduces the locality of the method. Although continuous spectral element methods parallelize quite well [29], their insistence on continuity makes them cumbersome to employ in either a nonconforming approach [19] or in an adaptive solution strategy.

In this paper we introduce a nodal high-order discontinuous Galerkin method for geophysical flows on the sphere. Like continuous spectral element methods, discontinuous Galerkin methods (DGM) can be constructed to have high-order accuracy, while maintaining a large degree of locality, hence enabling high parallel performance and adaptive solution procedures. The locality of these methods ensures that they can be used with any type of grid, e.g., unstructured and nonconforming if needed. In this paper we shall demonstrate this by using unstructured icosahedral grids.

As a first step toward the construction of a fully 3D atmospheric model, we demonstrate the efficiency and accuracy of the nodal high-order DGM by considering the shallow water equations on the sphere. The shallow water equations contain all of the horizontal operators required in an atmospheric model and thus represent a good first test for newly proposed methods for atmospheric models.

The remainder of the paper is organized as follows. In Section 2 we introduce the spherical shallow water equations and discuss the reasons for using a Cartesian grid. This sets the stage for Section 3, which introduces the numerical scheme and discusses in detail the spatial curvilinear representation of the solution as well as the formulation that enables one to satisfy the equations in a discontinuous fashion. We also discuss the temporal time-stepping scheme and the approach taken to generate a suitable grid on the sphere. In Section 4 we demonstrate the accuracy, efficiency, and robustness of the complete scheme for the solution of benchmark problems for the spherical shallow water equations. Section 5 contains a few remarks and outlines natural extensions of the work presented here.

\section{SPHERICAL SHALLOW WATER EQUATIONS}

The spherical shallow water equations in conservation form are given as

$$
\frac{\partial \boldsymbol{q}}{\partial t}+\nabla \cdot \boldsymbol{F}(\boldsymbol{q})=\boldsymbol{S}(\boldsymbol{x}, \boldsymbol{q})
$$

where

$$
\boldsymbol{q}=[\varphi, \varphi u, \varphi v, \varphi w]^{T}
$$


represents the state vector, $\boldsymbol{q}$, composed of the geopotential height, $\varphi$, and the three Cartesian velocity components, $(u, v, w)$, all being a function of $\boldsymbol{x} \in \mathrm{R}^{3}$ and time, $t$. The units for $\varphi$ and $\mathbf{u}$ are $(\mathrm{m} / \mathrm{s})^{2}$ and $\mathrm{m} / \mathrm{s}$, respectively.

The flux, $\boldsymbol{F}(\boldsymbol{q})$, takes the form

$$
\boldsymbol{F}(\boldsymbol{q})=\left[\begin{array}{c}
\varphi u \\
\varphi u^{2}+\frac{1}{2} \varphi^{2} \\
\varphi u v \\
\varphi u w
\end{array}\right] \hat{\boldsymbol{i}}+\left[\begin{array}{c}
\varphi v \\
\varphi u v \\
\varphi v^{2}+\frac{1}{2} \varphi^{2} \\
\varphi v w
\end{array}\right] \hat{\boldsymbol{j}}+\left[\begin{array}{c}
\varphi w \\
\varphi u w \\
\varphi v w \\
\varphi w^{2}+\frac{1}{2} \varphi^{2}
\end{array}\right] \hat{\boldsymbol{k}}
$$

where $(\hat{\boldsymbol{i}}, \hat{\boldsymbol{j}}, \hat{\boldsymbol{k}})$ represent the Cartesian unit vectors. The source term, $\boldsymbol{S}(\boldsymbol{x}, \boldsymbol{q})$, in Eq. (1), acting only on the momentum equations, is given as

$$
\boldsymbol{S}(\boldsymbol{x}, \boldsymbol{q})=-\frac{2 \Omega z \varphi}{R^{2}} \boldsymbol{x} \times \boldsymbol{u}-\varphi \nabla \varphi_{s}+\mu \boldsymbol{x}
$$

The first term in Eq. (3) accounts for the Coriolis force, with $R=6.371 \times 10^{6} \mathrm{~m}$ being the radius and $\Omega=7.292 \times 10^{-5} \mathrm{rad} / \mathrm{s}$ the angular velocity of the earth, while the second contribution models the effects of a variable surface height through the surface potential, $\varphi_{s}$. The last term is a Lagrange multiplier, the specification of which we shall return to shortly.

Equation (1) is derived from the incompressible and inviscid Navier-Stokes equations by vertically integrating the mass to yield the geopotential height equation (for further details see [24]). Contrary to most other work on the numerical solution of Eq. (1) on a spherical shell, we shall not recast it in spherical coordinates but rather maintain the Cartesian coordinates. The main motivation for doing so, albeit at the expense of introducing an additional momentum equation, is to avoid the problems associated with the polar singularity. For a spherical shell, described by the coordinates $(\lambda, \theta)$, of radius $R$ the divergence of a vector field, $\boldsymbol{F}=f \hat{\boldsymbol{\lambda}}+g \hat{\boldsymbol{\theta}}$, is given as

$$
\nabla \cdot \boldsymbol{F}=\frac{1}{R \cos \theta}\left[\frac{\partial f}{\partial \lambda}+\frac{\partial g \cos \theta}{\partial \theta}\right] .
$$

At the poles, i.e., $\theta= \pm \pi / 2$, this is a source of numerical problems, caused by the specific formulation rather than the nature of the shallow water equations and its solutions. While the use of a local Cartesian coordinate system has been used to overcome these problems in the past [28] we have, guided by the results of previous work [10], chosen to maintain the Cartesian formulation everywhere. This Cartesian formulation poses no difficulty to the construction of a 3D atmospheric primitive equation model as shown in [11] for the continuous spectral element method.

To ensure that the fluid particles remain on the spherical shell, we require that the fluid velocity remains perpendicular to the position vector, i.e., $\boldsymbol{x} \cdot \boldsymbol{u}=0$, which yields the Lagrange multiplier

$$
\mu(\boldsymbol{x}, \boldsymbol{q})=\frac{1}{R^{2}}\left[\varphi \boldsymbol{x} \cdot \nabla \varphi_{s}+\boldsymbol{x} \cdot(\nabla \cdot \tilde{\boldsymbol{F}})\right]
$$

Here $\tilde{\boldsymbol{F}}$ represents the parts of the flux, Eq. (2), associated with the momentum equations, i.e., the last three fluxes. As no constraint is needed on the geophysical potential, essentially representing the local mass, the multiplier is needed in the momentum equations only. 


\section{THE NUMERICAL SCHEME}

In developing the numerical scheme for the solution of Eq. (1), we shall split the discussion into a treatment of the spatial discretization and the approximation of the resulting semidiscrete approximation.

\subsection{The Spatial Approximation}

The discussion of the spatial approximation scheme involves, as does any development of a scheme for solving partial differential equations, attention to the questions of how to represent the solution as well as in which way the equations are required to be satisfied. In the following we address these two issues in more detail.

\subsubsection{Representing the Solution and Basic Operations}

Initially, we assume that the computational domain, S, i.e., a spherical shell, is covered by $K$ nonoverlapping curvilinear quadrilaterals, $\mathrm{D}$, such that

$$
\mathrm{S}=\bigcup_{k=1}^{K} \mathrm{D}^{k}
$$

The construction of this sphere covering is not entirely trivial and we shall return to this problem in Section 4. For now, however, we simply assume its existence.

To enable the efficient and accurate computation of operations such as differentiation and integration, we introduce a nonsingular mapping, $\boldsymbol{x}=\boldsymbol{\Psi}(\boldsymbol{\xi})$, which connects the local physical coordinates, $\boldsymbol{x}=(x, y, z)$, defined on $\mathrm{D}$ with a reference system $\boldsymbol{\xi}=(\xi, \eta, \zeta)$, defined on the local element such that $(\xi, \eta)$ lies on the spherical surface. Thus, $\zeta$ represents the spherical radius vector itself, i.e., $\zeta$ constant corresponds to a shell of a constant radius. For simplicity we assume that $(\xi, \eta) \in[-1,1]^{2}$ on each element, see Fig. 1.

Associated with the local mapping, $\Psi$, is the transformation Jacobian, $J=\frac{\partial x}{\partial \xi}$, and the determinant

$$
|J|=\frac{\partial \boldsymbol{x}}{\partial \zeta} \cdot \boldsymbol{G}, \quad \boldsymbol{G}=\frac{\partial \boldsymbol{x}}{\partial \xi} \times \frac{\partial \boldsymbol{x}}{\partial \eta},
$$

where $\boldsymbol{G}$ represents the surface conforming component of the mapping (see [10] for further details).

The mapping also supplies the local metric, $\nabla \xi$ and $\nabla \eta$, as well as the local normal vectors at the surface of the element. Indeed, as illustrated in Fig. 1, the normals are given as

$$
\hat{\boldsymbol{n}}=\left.\eta \frac{\nabla \eta}{|\nabla \eta|}\right|_{\eta= \pm 1}, \quad \hat{\boldsymbol{n}}=\left.\xi \frac{\nabla \xi}{|\nabla \xi|}\right|_{\xi= \pm 1},
$$

for the sides $1 / 3$ and $2 / 4$, respectively.

With this in place we can now focus on the local element-wise representation of the solution, $\boldsymbol{q}$, and the approximation of operations such as differentiation and integration. For simplicity, we assume $\zeta$ to be unity in what remains and denote $\xi=(\xi, \eta)$ unless clarification is deemed necessary. 


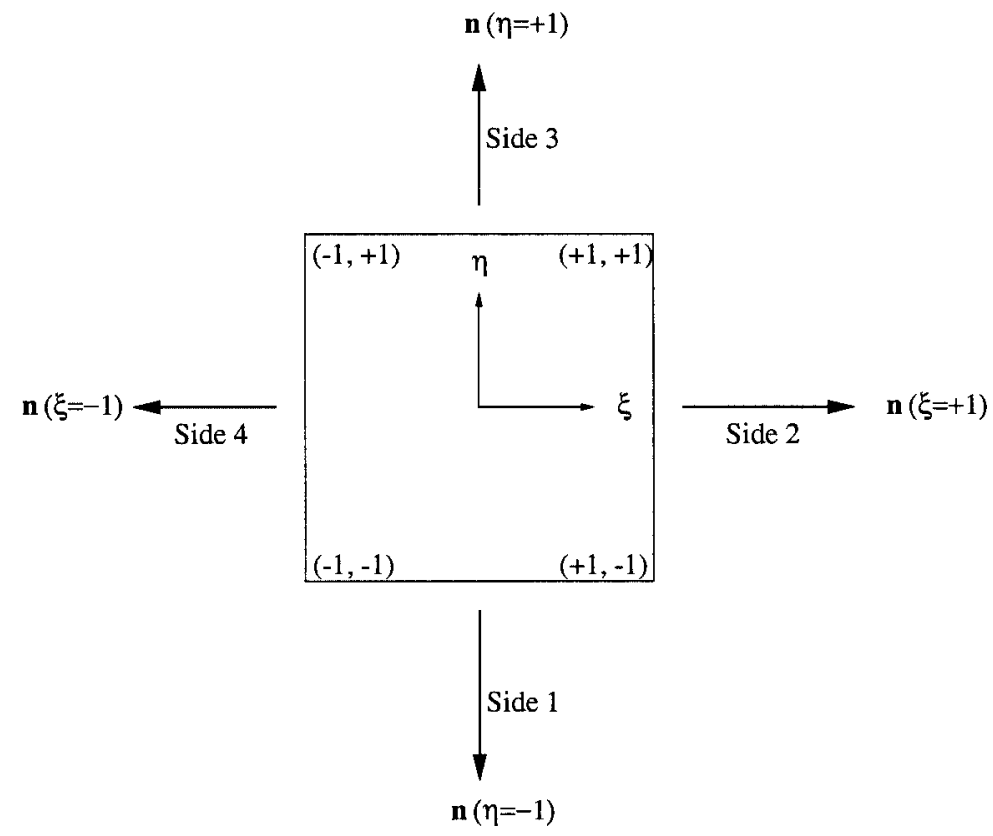

FIG. 1. The geometry of the mapping and the associated metric information.

The simple structure of the standard element, I, spanned by $\xi \in[-1,1]^{2}$, makes it natural to represent the local solution by an $N$ th order polynomial in $\boldsymbol{\xi}$ as

$$
\boldsymbol{q}_{N}(\boldsymbol{x})=\sum_{k=1}^{(N+1)^{2}} \boldsymbol{q}_{N}\left(\boldsymbol{x}_{k}\right) L_{k}(\boldsymbol{x}),
$$

where $\boldsymbol{x}_{k}$ represents $(N+1)^{2}$ grid points and $L_{k}(\boldsymbol{x})$ reflects the associated multivariate Lagrange interpolation polynomial. The logical square structure of I simplifies matters further in that we can express the Lagrange polynomial by a tensor-product as

$$
L_{k}(\boldsymbol{x})=h_{i}(\xi(\boldsymbol{x})) h_{j}(\eta(\boldsymbol{x})),
$$

where $i, j=0, \ldots, N$.

While many choices of the grid points, $\left(\xi_{i}, \eta_{j}\right)$, are possible, it is natural to choose the Legendre-Gauss-Lobatto points, given as the tensor-product of the roots of

$$
\left(1-\xi^{2}\right) P_{N}^{\prime}(\xi)=0
$$

where $P_{N}(\xi)$ is the $N$ th order Legendre polynomial. This choice is natural as these points are endowed with a Gaussian quadrature rule which shall become useful shortly. With this choice, the one-dimensional Lagrange polynomials, $h_{i}(\xi)$, also known as the Legendre cardinal functions, become

$$
h_{i}(\xi)=-\frac{1}{N(N+1)} \frac{\left(1-\xi^{2}\right) P_{N}^{\prime}(\xi)}{\left(\xi-\xi_{i}\right) P_{N}\left(\xi_{i}\right)},
$$

and likewise for $h_{j}(\eta)$. 
The choice of the Legendre-Gauss-Lobatto points enables the straightforward approximation of element-wise integrals, i.e.,

$$
\int_{\mathrm{D}} q(\boldsymbol{x}) d \boldsymbol{x}=\int_{\mathrm{I}} q(\boldsymbol{\xi}) J(\xi) d \boldsymbol{\xi} \simeq \sum_{i, j=0}^{N} q\left(\xi_{i}, \eta_{j}\right) J\left(\xi_{i}, \eta_{j}\right) \omega_{i}^{\xi} \omega_{j}^{\eta}
$$

where $J$ represents the local Jacobian for the transformation between D and I, and $\omega_{i}^{\xi}$ and $\omega_{j}^{\eta}$ are the Gaussian quadrature weights,

$$
\omega_{i}^{\xi}=\frac{2}{N(N+1)}\left(\frac{1}{P_{N}\left(\xi_{i}\right)}\right)
$$

associated with the one-dimensional Legendre-Gauss-Lobatto quadrature. We recall that if $q J$ is a polynomial of at most degree $2 N-1$ in each local coordinate, the quadrature is exact.

The evaluation of surface integrals, made particularly simple by the natural separation between interior and edge (side) nodes in the nodal formulation, follows the same line of thinking, i.e.,

$$
\begin{aligned}
\oint_{\delta \mathrm{D}} q(\boldsymbol{x}) d \boldsymbol{x} & =\oint_{\delta \mathbf{l}} q(\boldsymbol{\xi}) J(\xi) d \boldsymbol{\xi} \\
\simeq & \sum_{i=0}^{N}\left[q\left(\xi_{i},-1\right) J\left(\xi_{i},-1\right)+q\left(\xi_{i}, 1\right) J\left(\xi_{i}, 1\right)\right] \omega_{i}^{\xi} \\
& +\sum_{j=0}^{N}\left[q\left(-1, \eta_{j}\right) J\left(-1, \eta_{j}\right)+q\left(1, \eta_{j}\right) J\left(1, \eta_{j}\right)\right] \omega_{j}^{\eta} .
\end{aligned}
$$

\subsubsection{Satisfying the Equation}

With the local representation of the solutions in place, we now proceed to consider the question of how to satisfy the equation. We assume that the solution, $\boldsymbol{q}$, to Eq. (1) is represented locally by high-order polynomials, $\boldsymbol{q}_{N}$, defined on the curvilinear quadrilateral, $\mathrm{D}$, and require that the equation be satisfied element-wise in the following discontinuous Galerkin way

$$
\int_{\mathrm{D}}\left(\frac{\partial \boldsymbol{q}_{N}}{\partial t}+\nabla \cdot \boldsymbol{F}_{N}-\boldsymbol{S}_{N}\right) L_{k}(\boldsymbol{x}) d \boldsymbol{x}=\oint_{\delta \mathrm{D}} L_{k}(\boldsymbol{x}) \hat{\boldsymbol{n}} \cdot\left[\boldsymbol{F}_{N}-\boldsymbol{F}_{N}^{*}\right] d \boldsymbol{x},
$$

where $L_{k}(\boldsymbol{x})\left(k \in\left[1, \ldots,(N+1)^{2}\right]\right)$ is the local polynomial basis, Eq. (5). We have also introduced the polynomial representation of the flux, $\boldsymbol{F}_{N}=\boldsymbol{F}_{N}\left(\boldsymbol{q}_{N}\right)$, and the source, $\boldsymbol{S}_{N}=\boldsymbol{S}_{N}\left(\boldsymbol{x}, \boldsymbol{q}_{N}\right)$, as

$$
\boldsymbol{F}_{N}\left(\boldsymbol{q}_{N}\right)=\sum_{k=1}^{(N+1)^{2}} \boldsymbol{F}\left(\boldsymbol{q}_{N}\left(\boldsymbol{x}_{k}\right)\right) L_{k}(\boldsymbol{x}), \quad \boldsymbol{S}_{N}\left(\boldsymbol{x}, \boldsymbol{q}_{N}\right)=\sum_{k=1}^{(N+1)^{2}} \boldsymbol{S}\left(\boldsymbol{x}_{k}, \boldsymbol{q}_{N}\left(\boldsymbol{x}_{k}\right)\right) L_{k}(\boldsymbol{x}) .
$$

The numerical flux, $\boldsymbol{F}_{N}^{*}$, in Eq. (6) shall be discussed in detail shortly. Prior to that, however, 
a few remarks concerning Eq. (6) are in order. First, we note that the interface conditions, introduced into the formulation through the numerical flux, are enforced only weakly, i.e., the solution is in general discontinuous. As we shall see, however, this does not impact the accuracy as the size of the jump vanishes to the order of the interior approximation. Furthermore, the discontinuous formulation ensures a highly parallel scheme as all communication is local between elements sharing edges in two dimensions and faces in three dimensions. Finally, the locality of the approximation makes it straightforward to extend the scheme to include support for different orders of approximations in different elements, nonconforming elements, or different types of elements, e.g., triangles and quadrilaterals.

Before discussing the numerical flux, let us note that a mathematically equivalent but numerically different formulation of Eq. (6) can be obtained by an integration by parts to recover

$$
\int_{\mathrm{D}}\left(\frac{\partial \boldsymbol{q}_{N}}{\partial t}-\boldsymbol{F}_{N} \cdot \nabla-\boldsymbol{S}_{N}\right) L_{k}(\boldsymbol{x}) d \boldsymbol{x}=-\oint_{\delta \mathrm{D}} L_{k}(\boldsymbol{x}) \boldsymbol{F}_{N}^{*} d \boldsymbol{x} .
$$

This can be recognized as the classical discontinuous Galerkin method for conservation laws $[1,2,5,20]$. To distinguish between the two formulations, we shall refer to Eq. (6) as the divergence form and the more familiar one, Eq. (7), as the Green's form. Other terms often used to describe these formulations are the strong and the weak form, respectively.

The numerical flux, $\boldsymbol{F}_{N}^{*}$, is the part of the formulation that allows information to be passed between the individual elements, the union of which forms $\mathrm{S}$. The discontinuous formulation implies that the solution at an interface is nonunique and we must ensure that an unique solution be identified and passed to both elements in a way consistent with the dynamics of the problem.

This is a situation similar to a classical finite volume formulation to which Eq. (7) reduces for the lowest order elements. Thus, we can borrow from the extensive literature devoted to the development and analysis of numerical fluxes within the context of finite volume methods, e.g., upwinding by linearization or approximate Riemann solvers such as Roe [25], Engquist-Osher [7], or Van Leer [30] fluxes.

For simplicity and generality we use subsequently the simple Lax-Friedrichs flux of the form

$$
\boldsymbol{F}_{N}^{*}=\frac{\boldsymbol{F}_{N}\left(\boldsymbol{q}_{N}\right)+\boldsymbol{F}_{N}\left(\boldsymbol{p}_{N}\right)}{2}-\frac{|\lambda|}{2}\left(\boldsymbol{p}_{N}-\boldsymbol{q}_{N}\right)
$$

where $\boldsymbol{q}_{N}$ refers to the local computed solution and $\boldsymbol{p}_{N}$ refers to the solution in the neighboring element. The dissipative term is scaled by $|\lambda|$, which represents the maximum (local) eigenvalue of the flux Jacobian

$$
\frac{\partial \hat{\boldsymbol{n}} \cdot \boldsymbol{F}}{\partial \boldsymbol{q}}=\left[\begin{array}{cccc}
0 & \hat{n}_{x} & \hat{n}_{y} & \hat{n}_{z} \\
\hat{n}_{x} \varphi-u U & \hat{n}_{x} u+U & \hat{n}_{y} u & \hat{n}_{z} u \\
\hat{n}_{y} \varphi-v U & \hat{n}_{x} v & \hat{n}_{y} v+U & \hat{n}_{z} v \\
\hat{n}_{z} \varphi-w U & \hat{n}_{x} w & \hat{n}_{y} w & \hat{n}_{z} w+U
\end{array}\right]
$$


where

$$
U=\hat{\boldsymbol{n}} \cdot \mathbf{u} \text {. }
$$

The eigenvalues of the flux Jacobian are $\Lambda=[U, U, U+\sqrt{\varphi}, U-\sqrt{\varphi}]^{T}$, such that

$$
|\lambda|=|U|+\sqrt{\varphi}
$$

is the maximum wave speed of the shallow water equations entering the Lax-Friedrichs flux. While it is well known that the use of a Lax-Friedrichs flux in a classical finite volume formulation leads to a very dissipative scheme, this is likewise known to be much less of a problem in a high-order formulation [6].

To simplify matters further for both formulations, let us introduce

$$
\mathbf{M}_{l k}=\int_{\mathrm{D}} L_{l}(\boldsymbol{x}) L_{k}(\boldsymbol{x}) d \boldsymbol{x}, \quad \mathbf{D}_{l k}=\int_{\mathrm{D}} L_{l}(\boldsymbol{x}) \nabla L_{k}(\boldsymbol{x}) d \boldsymbol{x},
$$

as the mass matrix and the differentiation operator, respectively. Note that $\mathbf{D}=\left[\mathbf{D}^{x}, \mathbf{D}^{y}, \mathbf{D}^{z}\right]$ is a vector of matrices corresponding to a discrete gradient operator. To account for the source we define

$$
\mathbf{S}_{l k}=\frac{2 \Omega}{R^{2}} \sum_{m, n=1}^{(N+1)^{2}} z_{m} \boldsymbol{x}_{n} \int_{\mathrm{D}} L_{m}(\boldsymbol{x}) L_{n}(\boldsymbol{x}) L_{l}(\boldsymbol{x}) L_{k}(\boldsymbol{x}) d \boldsymbol{x}
$$

and

$$
\mathbf{M}_{l k}^{\varphi}=\int_{\mathrm{D}} L_{l}(\boldsymbol{x}) L_{k}(\boldsymbol{x}) \nabla \varphi_{s}(\boldsymbol{x}) d \boldsymbol{x}
$$

where $\mathbf{D}, \mathbf{S}$, and $\mathbf{M}^{\varphi}$ are 3-vectors of matrices.

Finally, we introduce the operator associated with the surface integral as

$$
\mathbf{F}_{l k}=\oint_{\delta \mathrm{D}} L_{l}(\boldsymbol{x}) L_{k}(\boldsymbol{x}) d \boldsymbol{x}
$$

where $l$ includes the trace of nodes on the face of $D$ only. Denoting the local element-wise grid vector of the geopotential as $\varphi_{N}$, the three momentum components as $\boldsymbol{\varphi} \boldsymbol{u}_{N}$ and the corresponding state vector as $\boldsymbol{q}_{N}=\left[\boldsymbol{\varphi}_{N}, \boldsymbol{\varphi} \boldsymbol{u}_{N}\right]^{T}$, we can now express the semi-discrete approximation of the divergence form, Eq. (6), as

$$
\begin{aligned}
\left(\mathrm{I}_{4}\right. & \otimes \mathrm{M}) \frac{d}{d t} \boldsymbol{q}_{N}+\mathbf{D} \cdot \boldsymbol{F}_{N}\left(\boldsymbol{q}_{N}\right) \\
& =-\left[\begin{array}{cc}
0 & 0 \\
\mathbf{M}^{\varphi} & \mathbf{S} \times
\end{array}\right] \boldsymbol{q}_{N}+\left[\begin{array}{cc}
0 & 0 \\
0 & \mu \mathrm{I}_{3} \otimes \mathrm{M}
\end{array}\right]\left[\begin{array}{c}
0 \\
\boldsymbol{x}_{N}
\end{array}\right]+\mathrm{I}_{4} \otimes \mathrm{F}\left[\boldsymbol{F}_{N}\left(\boldsymbol{q}_{N}\right)-\boldsymbol{F}^{*}\right],
\end{aligned}
$$

where $\mathrm{I}_{r}$ is a rank-r identity matrix and the Lagrange multipliers are contained in the diagonal matrix, $\mu=\operatorname{diag}\left[\mu_{1}, \ldots, \mu_{(N+1)^{2}}\right]$. Finally, we have introduced the arrays of nodal physical coordinates, $\boldsymbol{x}_{N}=\left[x_{N}, y_{N}, z_{N}\right]^{T}$. 
Similarly, we can express the Green's form, Eq. (7), using the above notation as

$\left(\mathrm{I}_{4} \otimes \mathrm{M}\right) \frac{d}{d t} \boldsymbol{q}_{N}-\mathbf{D}^{T} \cdot \boldsymbol{F}_{N}\left(\boldsymbol{q}_{N}\right)=-\left[\begin{array}{cc}0 & 0 \\ \mathbf{M}^{\varphi} & \mathbf{S} \times\end{array}\right] \boldsymbol{q}_{N}+\left[\begin{array}{cc}0 & 0 \\ 0 & \mu \mathrm{I}_{3} \otimes \mathbf{M}\end{array}\right]\left[\begin{array}{c}0 \\ \boldsymbol{x}_{N}\end{array}\right]-\left(\mathrm{I}_{4} \otimes \mathrm{F}\right) \boldsymbol{F}^{*}$

In both cases we can compute the Lagrange multipliers in a way similar to the continuous case, Eq. (4), i.e., by requiring that the discrete momentum is pointwise normal to the position vector.

\subsubsection{Communication on Distributed-Memory Computers}

It is worth emphasizing that both schemes given above are fully explicit in time, i.e., in contrast to a classical finite element/spectral element scheme no global assembly is required and the formulation is parallel by construction. As an example of the impact of this, consider an icosahedral grid based on quadrilaterals. Each element has only four edge neighbors but as many as nine vertex neighbors. This disparity between edge and vertex neighbors gets significantly worse in three dimensions. Therefore, although the length of the communication message for both the SEM and DGM formulations are equal, $\mathcal{O}(4 N+2)$ corresponding to the edge nodes, the communication requirements of the two schemes are very different as the SEM formulation has to communicate this message to at least twice as many neighbors as in the DGM formulation.

The only overhead associated with the DGM formulation is the additional memory required to store the multiple solutions at the overlapping regions of the elements, i.e., the edges (sides). For high-order elements, this memory overhead is clearly negligible as the number of edge to volume nodes decreases rapidly as the order increases.

\subsection{Temporal Integration and Stability}

The explicit nature of the semi-discrete formulation

$$
\frac{\partial \boldsymbol{q}_{N}}{\partial t}=\mathrm{B}\left(\boldsymbol{q}_{N}\right)
$$

where B signifies the operators given in Eqs. (9) and (10), makes it natural to use a standard explicit Runge-Kutta scheme. This yields

$$
\forall k=1, \ldots, 3: \boldsymbol{q}_{N}^{k+1}=\boldsymbol{q}_{N}^{k}+\Delta t \alpha_{k} \mathrm{~B}\left(\boldsymbol{q}^{k}\right)
$$

with $\boldsymbol{q}_{N}^{k=1}=\boldsymbol{q}_{N}^{n}$ and

$$
\boldsymbol{q}_{N}^{n+1}=\boldsymbol{q}_{N}^{n}+\frac{\Delta t}{6} \sum_{k=1}^{4} \beta_{k} \mathrm{~B}\left(\boldsymbol{q}^{k}\right)
$$

where

$$
\alpha_{1}=\alpha_{2}=\frac{1}{2}, \quad \alpha_{3}=1, \quad \text { and } \quad \beta_{1}=\beta_{4}=1, \quad \beta_{2}=\beta_{3}=2
$$


The time step, $\Delta t$, is chosen in order to ensure a stable scheme and will generally scale like

$$
\Delta t \leq \mathrm{CFL} \times \min _{\boldsymbol{x} \in \mathrm{D}}[|\boldsymbol{u} \cdot \chi|+\varphi \sqrt{\chi \cdot \chi}]^{-1}
$$

where the local grid distortion is measured by

$$
\chi=\left(\frac{\left|\xi_{x}\right|}{\Delta \xi}+\frac{\left|\eta_{x}\right|}{\Delta \eta}, \frac{\left|\xi_{y}\right|}{\Delta \xi}+\frac{\left|\eta_{y}\right|}{\Delta \eta}, \frac{\left|\xi_{z}\right|}{\Delta \xi}+\frac{\left|\eta_{z}\right|}{\Delta \eta}\right) .
$$

Here $(\Delta \xi, \Delta \eta)$ reflects the local average grid size and $\boldsymbol{u}=(u, v, w)$ the local velocity.

Even with a suitably chosen value of the time step it is well known that high-order methods are prone to instabilities due to the nonlinear mixing and lack of dissipation; see, e.g., [12]. This is particularly true for problems with marginally resolved phenomena where the nonlinear mixing of the solution with the Gibbs oscillations can drive the instability.

The standard approach to avoid this instability in a controlled manner is through the use of a weak high-order filter which modifies the high frequency modes without altering the well-resolved low frequency modes. As has been shown over the last decade, such filtering can be applied without sacrificing spectral accuracy [12, 28].

We shall focus on the filters developed by Boyd [3] and Vandeven [31]. While these filters perform well, their use is known to pose difficulties in a classical spectral element scheme where the solution is required to be continuous. As we shall see shortly, however, these difficulties vanish in the current formulation.

Following the discussion in [3], consider the state variables, $\boldsymbol{q}_{N}$, as

$$
\boldsymbol{q}_{N}(\boldsymbol{\xi})=\sum_{k=1}^{(N+1)^{2}} \boldsymbol{q}_{N}\left(\boldsymbol{\xi}_{k}\right) L_{k}(\boldsymbol{\xi})=\sum_{i, j=0}^{N} \hat{\boldsymbol{q}}_{i j} P_{i}(\xi) P_{j}(\eta)
$$

where $L_{k}(\boldsymbol{\xi})$ is the Lagrange polynomial associated with the points, $\boldsymbol{\xi}_{k} ; P_{i}(\xi)$ and $P_{j}(\eta)$ the Legendre polynomials in $\xi$ and $\eta$, respectively; and $\hat{\boldsymbol{q}}_{i j}$ the discrete Legendre expansion coefficients of the state vector computed by using the Legendre quadrature.

The filtering approach proposed in [3] involves the weighted sum

$$
\boldsymbol{q}_{N}^{F}=(1-v) \boldsymbol{q}_{N}+v \sum_{i, j=0}^{N} \sigma_{i} \sigma_{j} \hat{\boldsymbol{q}}_{i j} P_{i}(\xi) P_{j}(\eta),
$$

where $v$ is the filter weighting, i.e., $v=0$ represents no filtering and $v=1$ full filtering; typically, $v=0.2$ is used (see [28]). Furthermore,

$$
\sigma_{i}= \begin{cases}1 & \text { for } i<s \\ \sigma\left(\frac{i-s}{N-s}\right) & \text { for } s \leq i \leq N\end{cases}
$$

is the filter function with $\sigma$ being the Boyd-Vandeven filter [3] and $i=s, \ldots, N$ are the modes affected by the filter; $\sigma_{j}$ is defined likewise. Note that the filtering is performed in an element sense. In other words, each element yields its very own set of filtered values. In a spectral element formulation, continuity of the state vector across element edges must be enforced. As the filtering gives different values for grid points shared by adjacent elements, 
some form of weighting is typically required to recover continuity. With a discontinuous element formulation, however, this correction is unnecessary, hence greatly simplifying the inclusion of filters into the schemes.

\section{GENERATION OF ICOSAHEDRAL GRIDS ON THE SPHERE}

Contrary to the more traditional solution techniques exploiting spherical harmonics [13, $16,18,26,27]$, the use of a multielement formulation allows for the use of any type of grid, e.g., not only unstructured grids but also nonconforming grids.

The generation of the grid on the sphere is a challenging problem and in this section we describe the procedure for constructing the general high-order icosahedral grid proposed in $[9,10]$. This grid is derived from the icosahedron comprising 20 equilateral triangular elements and 12 grid points.

To construct icosahedral grids, we consider the initial icosahedron and subdivide each of the initial triangles by a triangular Lagrange polynomial of order $n_{I}$. Prior to mapping these elements onto the sphere, it is convenient to map the triangles onto a gnomonic space. The most unbiased mapping is obtained by mapping about the centroid of the triangles.

Let $\left(\lambda_{c}, \theta_{c}\right)$ be the centroid of the triangle we wish to map. The gnomonic mapping is then given by

$$
\begin{aligned}
& x=\frac{a \cos \theta \sin \left(\lambda-\lambda_{c}\right)}{\sin \theta_{c} \sin \theta+\cos \theta_{c} \cos \theta \cos \left(\lambda-\lambda_{c}\right)}, \\
& y=\frac{a\left[\cos \theta_{c} \sin \theta-\sin \theta_{c} \cos \theta \cos \left(\lambda-\lambda_{c}\right)\right]}{\sin \theta_{c} \sin \theta+\cos \theta_{c} \cos \theta \cos \left(\lambda-\lambda_{c}\right)} .
\end{aligned}
$$

To simplify matters a bit, we first apply a rotation whereby Eq. (13) becomes

$$
x=a \tan \lambda^{\prime}, \quad y=a \tan \theta^{\prime} \sec \lambda^{\prime},
$$

in the new coordinate system with the coordinates $(\lambda, \theta)$ located at $(0,0)$. The rotation mapping is given as

$$
\begin{aligned}
\lambda^{\prime} & =\arctan \left[\frac{\cos \theta \sin \left(\lambda-\lambda_{c}\right)}{\sin \theta_{c} \sin \theta+\cos \theta_{c} \cos \theta \cos \left(\lambda-\lambda_{c}\right)}\right], \\
\theta^{\prime} & =\arcsin \left[\cos \theta_{c} \sin \theta-\sin \theta_{c} \cos \theta \cos \left(\lambda-\lambda_{c}\right)\right] .
\end{aligned}
$$

This approach enables the construction of a general icosahedral grid defined by

$$
\begin{aligned}
N_{p}^{T} & =10\left(n_{I}-1\right)^{2}+20\left(n_{I}-1\right)+12, \\
N_{e}^{T} & =2\left(N_{p}^{T}-2\right), \\
N_{s}^{T} & =3\left(N_{p}^{T}-2\right),
\end{aligned}
$$

where $N_{p}^{T}, N_{e}^{T}$, and $N_{s}^{T}$ denote the number of points, elements, and sides, respectively, comprising the triangular grid.

Once the triangular icosahedral grid is constructed, we subdivide each triangular element into three quadrilateral elements. Upon dividing the triangles into quadrilaterals, one can 
construct the higher order collocation points inside each element resulting in a quadrilateral grid with the properties

$$
\begin{aligned}
& N_{p}=6\left(N_{p}^{T}-2\right) N^{2}+2, \\
& N_{e}=6\left(N_{p}^{T}-2\right), \\
& N_{s}=12\left(N_{p}^{T}-2\right),
\end{aligned}
$$

where $N_{p}, N_{e}$, and $N_{s}$ denote the number of points, elements, and sides comprising the quadrilateral grid. $N$ is the polynomial order used in the semi-discrete discontinuous Galerkin scheme discussed in Section 3.1.2.

Substituting the values in Eq. (16) into Eq. (17) yields

$$
\begin{aligned}
& N_{p}=60\left(n_{I}\right)^{2} N^{2}+2, \\
& N_{e}=60\left(n_{I}\right)^{2} \\
& N_{s}=120\left(n_{I}\right)^{2} .
\end{aligned}
$$

Table I provides examples of grids for various values of $n_{I}$ and $N$. Examples of corresponding grids for $n_{I}=1$ and $N=4,8,16$, and 32 are illustrated in Fig. 2.

\section{TABLE I}

The Number of Grid Points, Elements, and Sides for the Icosahedral Grid as a Function of $n_{I}$ and $N$

\begin{tabular}{rrrrrr}
\hline$n_{I}$ & $N$ & $n_{I} \cdot N$ & \multicolumn{1}{c}{$N_{p}$} & \multicolumn{1}{c}{$N_{e}$} & \multicolumn{1}{c}{$N_{s}$} \\
\hline 1 & 4 & 4 & 962 & 60 & 120 \\
1 & 8 & 8 & 3842 & 60 & 120 \\
1 & 16 & 16 & 15362 & 60 & 120 \\
1 & 32 & 32 & 61442 & 60 & 120 \\
1 & 64 & 64 & 245762 & 60 & 120 \\
4 & 1 & 4 & 962 & 960 & 1920 \\
8 & 1 & 8 & 3842 & 3840 & 7680 \\
16 & 1 & 16 & 15362 & 15360 & 30720 \\
32 & 1 & 32 & 61442 & 61440 & 122880 \\
64 & 1 & 64 & 245762 & 245760 & 491520 \\
2 & 2 & 4 & 962 & 240 & 480 \\
4 & 2 & 8 & 3842 & 960 & 1920 \\
8 & 2 & 16 & 15362 & 3840 & 7680 \\
16 & 2 & 32 & 61442 & 15360 & 30720 \\
32 & 2 & 64 & 245762 & 61440 & 122880 \\
1 & 4 & 4 & 962 & 60 & 120 \\
2 & 4 & 8 & 3842 & 240 & 480 \\
4 & 4 & 16 & 15362 & 960 & 1920 \\
8 & 4 & 32 & 61442 & 3840 & 7680 \\
16 & 4 & 64 & 245762 & 15360 & 30720 \\
\hline
\end{tabular}


a)

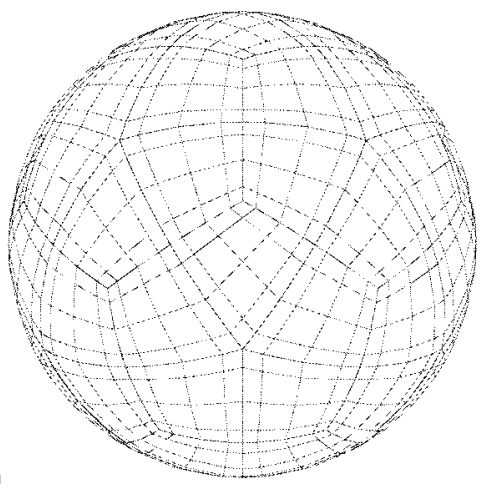

c)

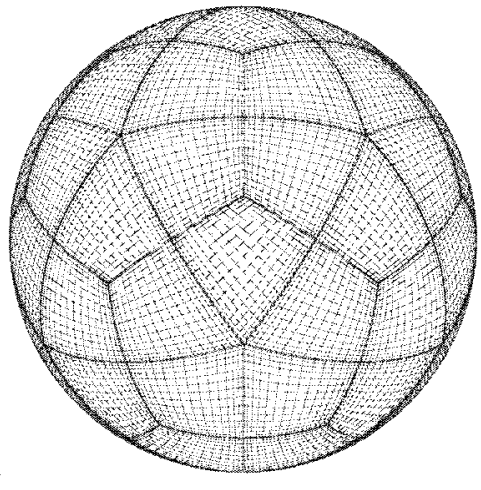

b)

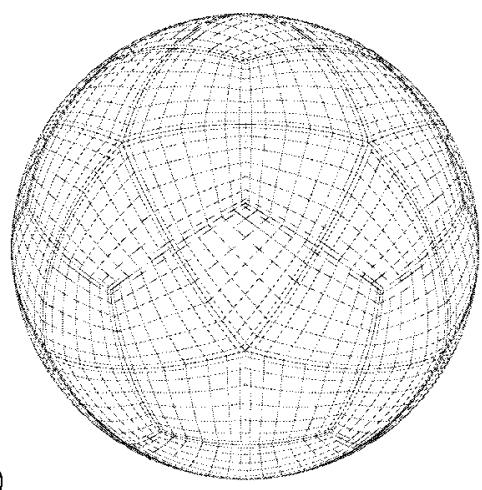

d)

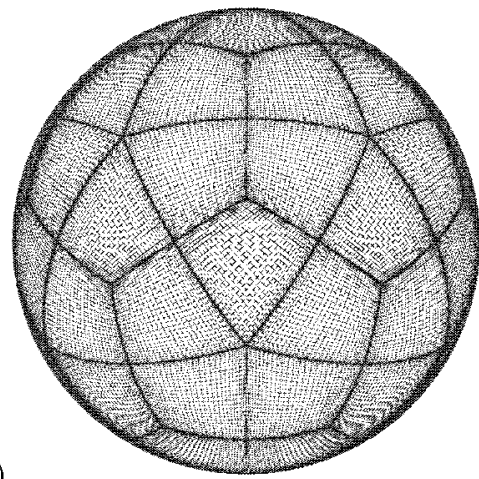

FIG. 2. An icosahedral grid for $n_{I}=1$ and a) $N=4$, b) $N=8$, c) $N=16$, and d) $N=32$.

\section{RESULTS}

In the following we evaluate the performance of the scheme discussed above. As a measure of the error, we use the relative $L_{2}$ error

$$
\left\|\boldsymbol{q}_{N}\right\|_{L_{2}}=\sqrt{\frac{\int_{\mathrm{D}}\left(\boldsymbol{q}_{\mathrm{exact}}-\boldsymbol{q}_{N}\right)^{2} d \boldsymbol{x}}{\int_{\mathrm{D}} \boldsymbol{q}_{\mathrm{exact}}^{2} d \boldsymbol{x}}} .
$$

Here $\boldsymbol{q}_{N}$ represents the computed state variables and $\boldsymbol{q}_{\text {exact }}$ the exact values when available. The global error is computed as a broken norm using the local quadratures.

Seven test cases are used in order to test the algorithms and form a framework for comparison between the two formulations. Cases 1, 2, 3, 5 and 6 correspond to the test cases given in [32]. Case 4 has been used as a test case for the shallow water equations in $[9,10,22,23]$. Cases 1,2 , and 3 have analytic solutions and are used to evaluate the accuracy of the discontinuous Galerkin method quantitatively. Cases 4, 5, and 6, on the other hand, do not have analytic solutions and are thus used to obtain a qualitative assessment of the accuracy of the scheme. In addition, Case 7 is used to test our scheme on flows containing nonsmooth or underresolved solutions. For this test case we compare our nodal DGM scheme with the continuous spectral element method in [10].

- Case 1: Steady-State Advection. This case concerns the solid body rotation of a cosine wave. It only tests the mass equation as the velocity field is assumed to remain unchanged 
throughout the computation. The cosine wave completes one full revolution after 12 days of integration.

- Case 2: Global Steady-State Nonlinear Zonal Geostrophic Flow. This case is a steadystate solution to the nonlinear shallow water equations. The equations are geostrophically balanced and remain so for the duration of the integration. The velocity field thus remains constant throughout the computation. The geopotential height $\varphi$ undergoes a solid body rotation but since the initial height field is given as a constant band, the solution remains the same throughout the time integration. The velocity field is the same as that used in Case 1 . Williamson et al. [32] recommend that the error be computed after five days of integration.

- Case 3: Steady-State Nonlinear Zonal Geostrophic Flow with Compact Support. This case is similar to Case 2 except that the velocity is zero everywhere except in a very small isolated region. This isolated region, or jet, encapsulates the flow and limits the geopotential height field to remain within a very confined circular region. As in Case 2, the errors are computed after five days.

- Case 4: Dancing High-Low Waves. This case comes from [22] and is not an analytic solution to the shallow water equations. The initial geopotential height comprises two large waves with the left wave being the low wave and the right wave being the high wave, when viewed from the north pole. The waves rotate clockwise in a swirling dance-like fashion. After 10 days of integration, the low wave is again on the left and the high wave is on the right.

- Case 5: Zonal Flow over an Isolated Mountain. This case is similar to Case 2 except that a mountain has been included. This is the only problem in the test cases studied here which includes topography. The mountain is conical in shape and forces the zonal flow to deflect off the mountain. Due to the zonal flow impinging on the mountain, wave structures form and propagate around the sphere. Results are reported for a 10-day integration period.

- Case 6: Rossby-Haurwitz Wave. Although Rossby-Haurwitz waves are not analytic solutions to the shallow water equations, they have become a standard test case. In a nondivergent barotropic model, the initial geopotential height field undergoes a solid body rotation in a counterclockwise direction (when viewed from the north pole). Results are reported for a 14-day integration.

- Case 7: Steady-State Advection of a Circular Column. This case is similar to Case 1 except that the mass, $\varphi$, is now assumed to be a circular column with values $\varphi \in[0,100]$. This circular column is the 2D analog of the 1D square wave and thus no longer represents a smooth solution. We present this case in order to judge qualitatively the performance of our nodal DGM scheme when confronted with steep gradients.

In the following we shall use these seven benchmarks as the stick against which to measure the scheme and its numerical properties such as robustness and accuracy. For the former we shall discuss the impact of various simplifications and approximations introduced into the two schemes, Eqs. (9) and (10), while the latter is evaluated by comparison with exact solutions as well as studies of a more qualitative nature. Throughout this section, unless explicitly stated, no filtering is used.

\subsection{Basic Convergence Tests}

To discuss and illustrate the advantages of using a high-order scheme for solving the shallow water equations we examine the solution of Cases 1, 2, and 3 using the divergence form of the scheme, Eq. (9), only. 
Figure 3 shows the computed mass error for the three cases using the following orders of accuracy: $N=1$ (dashed), $N=2$ (dotted), $N=4$ (dashed-dotted), and $n_{I}=1$ (solid). We plot the mass error norm as a function of the product $n_{I} N$. The $N=1$ results are obtained by using linear elements but increasing $n_{I}$ for increasing $n_{I} N$, i.e., it corresponds to a classic element refinement known as $h$-refinement. In contrast, the $n_{I}=1$ results shown in the plots represent our $N$ th order scheme where we keep the number of elements constant (given by $n_{I}=1$ ) and increase $N$ as is done in classic high-order/spectral convergence.

The results in Fig. 3 confirm the spectral accuracy of the scheme. These results also illustrate well the advantages offered by high-order schemes over low-order schemes in terms of accuracy.

However, it is crucial to understand the cost incurred by this additional accuracy. If the high-order scheme is prohibitively expensive for levels of accuracy of relevance, then the scheme loses much of its appeal.

In order to measure cost versus accuracy, we consider the number of operations associated with the different schemes. The simplest reasonable operation count is $\mathcal{O}\left(N^{5} N_{e}^{3 / 2}\right)$, where $N$ is the local approximation order on the $N_{e}$ elements. This estimate is obtained by including the $N_{e}$ evaluations of the derivatives, being $\mathcal{O}\left(N^{3} N_{e}\right)$, and including an additional $\mathcal{O}\left(N^{2} \sqrt{N_{e}}\right)$ work to account for the number of time steps required to advance the high-order method. Taking the expression for $N_{e}$ from Eq. (20), this yields an estimate of the work as $\mathcal{O}\left(N^{5} N_{I}^{3}\right)$.

The main interest is to limit the number of operations needed to achieve a result with a given accuracy; this will vary depending on the problem. Let us first consider the results of Case 1, illustrated in Fig. 3a. Taking an accuracy of $10^{-3}$ as the goal, Fig. 3a shows that the fourth-order scheme is the one with the least operations, about half that of the second-order scheme and almost five times less than the first-order scheme. In a similar fashion, one finds for Cases 2 and 3, and an accuracy goal of $10^{-6}$, that the $N$ th order scheme is most efficient.

These results provide only guidelines but they do confirm the advantages in using a high-order scheme over lower accuracy methods. Whether one should use the highest order approximation possible or rather limit the order and refine the element size may well be problem-dependent. However, as has also been found in other similar studies $[10,14,15$, $17,28]$, it is generally advantageous to use a moderate order of approximation, $N \in[8,16]$, and refine the element size accordingly to achieve a practical level of accuracy. Only for problems requiring very high accuracy or long time integration can one benefit from using a very high order scheme, i.e., $N>16$. The results obtained here support the validity of these guidelines.

\subsection{The Divergence Form versus the Green's Form}

As a second test we shall evaluate the differences between the divergence formulation, Eq. (9), and Green's formulation, Eq. (10), subject to various approximations and simplifications. As a basis for this comparison we shall again use Cases 1, 2, and 3. First, we examine the two formulations using full and diagonal mass matrices. Second, we examine both formulations using exact and inexact integration to compute the discrete operators. Although these simplifications and approximations appear to be minor, they have a significant impact on the performance of the divergence and Green's formulations. 

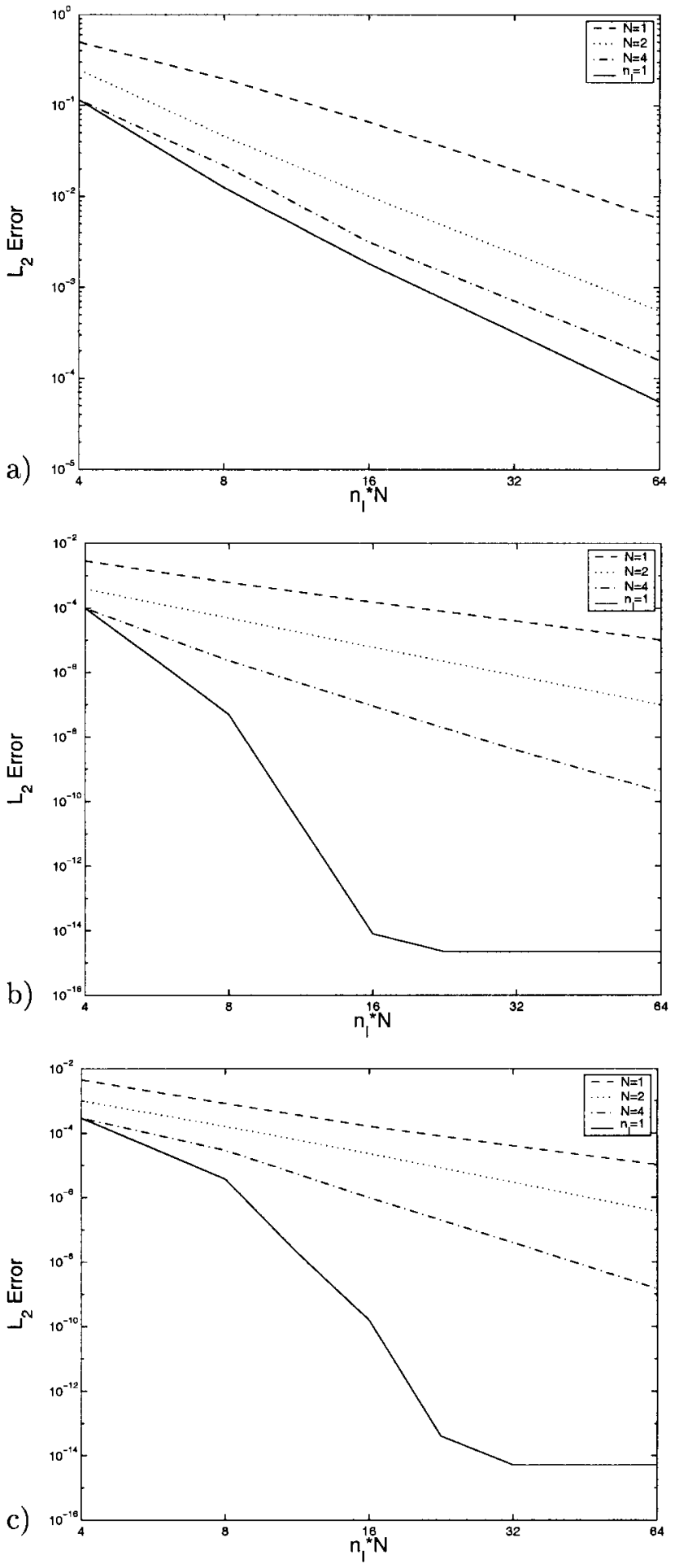

FIG. 3. The mass error norm for the divergence form of the DGM comparing low-order schemes $(N \leq 4)$ with the $N$ th order scheme $\left(n_{I}=1\right)$ for a) Case 1, b) Case 2, and c) Case 3 for a one-day integration. 


\subsubsection{Full versus Diagonal Mass Matrix}

One of the most immediate ways to improve the efficiency of the discontinuous Galerkin method is to approximate the mass matrix, M, Eq. (8), by a diagonal (or lumped) form. We form the diagonal approximation of the mass matrix by simply summing all of the entries of each row and storing the sum in the main diagonal.

Figure 4 shows the errors obtained using the full and diagonal mass matrices for the divergence and Green's forms, Eqs. (9) and (10), on the $n_{I}=1$ grid for Cases 1, 2, and 3. These results use exact numerical integration. These results show that there is little difference between using a full and diagonal mass matrix for both the divergence and Green's forms; only in Case 2 (Fig. 4b) does there seem to be a difference where the Green's form is adversely affected by this approximation.

\subsubsection{Exact versus Inexact Integration}

The evaluation of the discrete operators, e.g., Eq. (8), requires the computations of integrals. Despite the fact that we define the solution on Legendre-Gauss-Lobatto quadrature points, the curvilinear geometry, reflected by the transformation Jacobian, and the terms associated with the Coriolis force create polynomials of higher order and over-integration is needed to integrated these terms exactly. In fact, to integrate all of the matrices exactly requires $Q=(c N+1) / 2$ quadrature points, where $c$ is an integer constant denoting the factor of the maximum order matrix. For the spherical shallow water equations $c=4$, coming from the Coriolis term. For highly skewed elements, however, this factor increases to 6 because of the impact of the transformation Jacobians.

The question to address here is what is the effect of employing inexact integration only, as is traditionally done in continuous spectral element schemes [8, 17, 21, 28]. Here we shall simply use the straightforward quadrature associated with the Legendre-Gauss-Lobatto nodes, to evaluate the inner products and, thus, the discrete operators.

Figure 5 shows the mass error obtained using exact and inexact integration for the matrices of the divergence and the Green's forms on the $n_{I}=1$ grid for Cases 1, 2, and 3. Both the divergence and the Green's forms clearly lose accuracy when using inexact integration. However, the results indicate that the divergence form is more robust toward such approximations, i.e., the results obtained using the divergence form appear to be less affected by the approximations than those of the Green's form. This is most noticeable for Cases 2 and 3 (Figs. 5b and 5c, respectively) where the Green's form varies substantially between the exact and inexact integration.

\subsubsection{Divergence versus Green's Form}

The results in Figs. 4 and 5 indicate that the Green's form, Eq. (10), is superior in accuracy to the divergence form, Eq. (9), of the discontinuous Galerkin formulation if everything is done exactly, i.e., full mass matrices and over-integration to evaluate the discrete operators exactly. However, for all cases, the divergence form performs almost as well even when doing all operations exactly and it appears to be more robust to performance enhancing approximations such as diagonal mass matrices and inexact integration.

Based on these results we shall use the divergence form with inexact integration and diagonal mass matrices for the remainder of the paper as these approximations have a negligible impact on accuracy but a major impact on efficiency. It should be noted, however, 

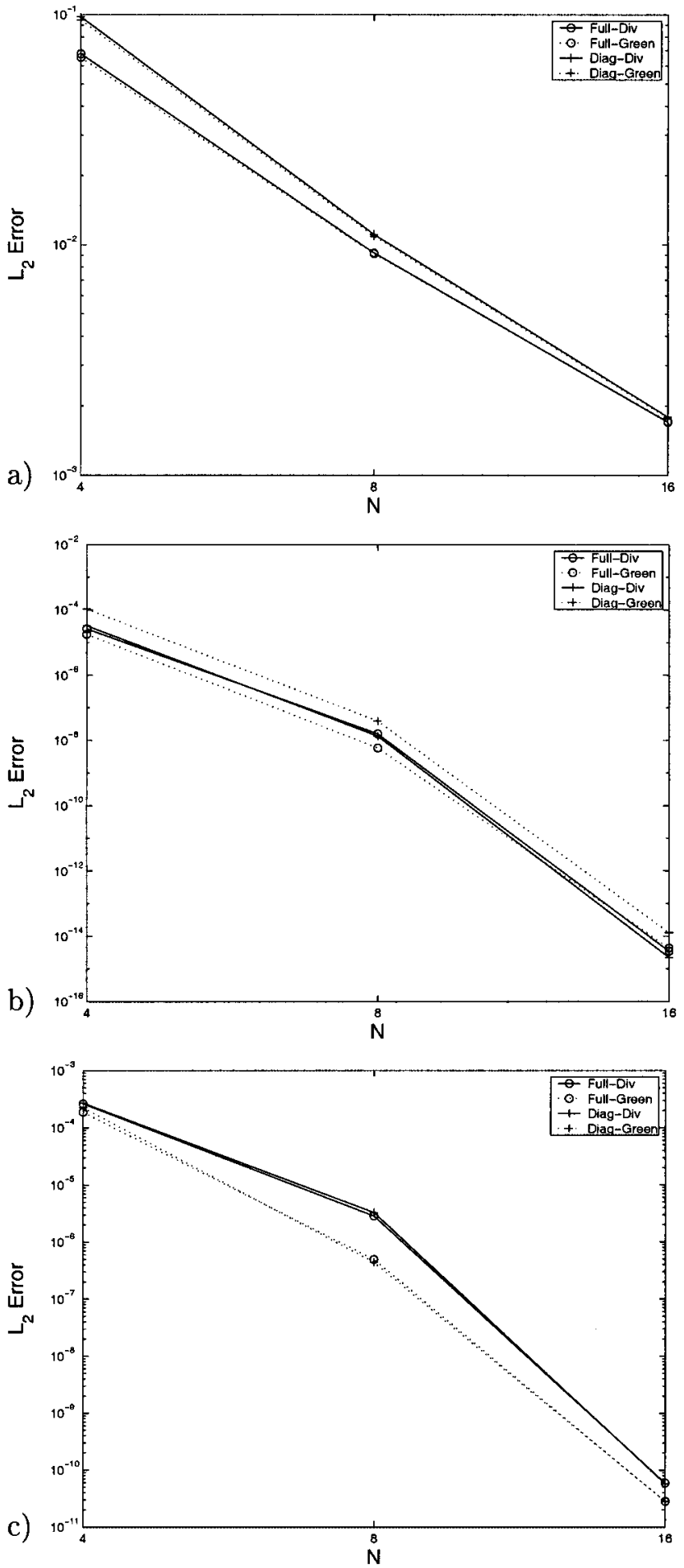

FIG. 4. Evaluation of the $\varphi$ error induced by using diagonal ( + ) and full $(\bigcirc)$ mass matrices, $M$, in the DGM formulations. The error norms are illustrated for the divergence form (solid line) and the Green's form (dotted line) for a) Case 1 b) Case 2, and c) Case 3. All results correspond to one day of integration. 

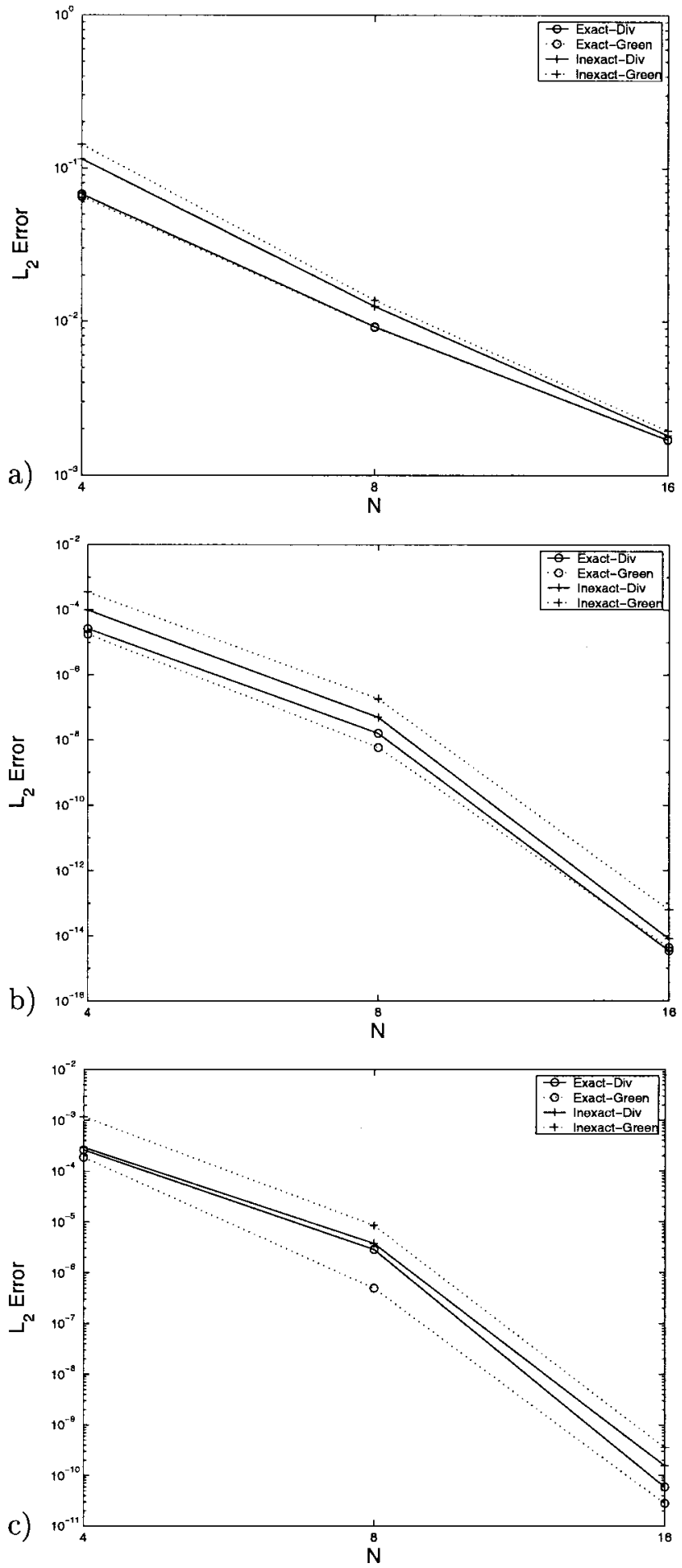

FIG. 5. Evaluation of the $\varphi$ error induced by using exact $(+)$ and inexact $(\bigcirc)$ integration for the computation of the operators in the DGM formulations. The error norms are illustrated for the divergence form (solid line) and the Green's form (dotted line) for a) Case, 1, b) Case 2, and c) Case 3. All results correspond to one day of integration. 
that the problems we have considered and on which we have based this choice are limited and it is not clear which formulation to choose for problems of a more general nature (e.g., problems with discontinuous solutions). We hope to address this in future work.

\subsection{Convergence and Stability Study}

In this section we show convergence results for all seven cases. Cases 1, 2, and 3 have analytic solutions and so we use these cases to judge the accuracy of our results quantitatively. Cases 4, 5, and 6, on the other hand, are only used to judge the convergence of the scheme qualitatively, as analytic solutions to these cases are unknown. Case 7 is used to judge the robustness of our scheme for nonsmooth solutions which are representative of steep fronts and other underresolved phenomena typically arising in the atmosphere. All seven test cases are integrated for long periods of time to validate the stability of the scheme. Cases 1, 2, and 3 require no filtering whereas Cases 4, 5, and 6 are filtered every 100 time steps. Results for Case 7 are shown both with and without filtering.

\subsubsection{Quantitative Study}

Figure 6 shows the mass error norm as a function of the approximation order, $N$, for Cases 1,2, and 3. The results for Case 1 are for a 12-day integration, which corresponds to a complete revolution of the cosine wave around the sphere. For Cases 2 and 3, the results are for 5-day integrations, which is the time frame recommended in [32].

For the latter two cases we observe exponential convergence rates until the effect of reaching machine double precision. Comparing these results to those published in [28] (Fig. 3 in that paper) we see that our nodal DGM model converges at a higher rate than their continuous spectral element model. In addition, comparing Table II of that paper we see that our nodal DGM model performs better than all the models listed in [28] for all three test

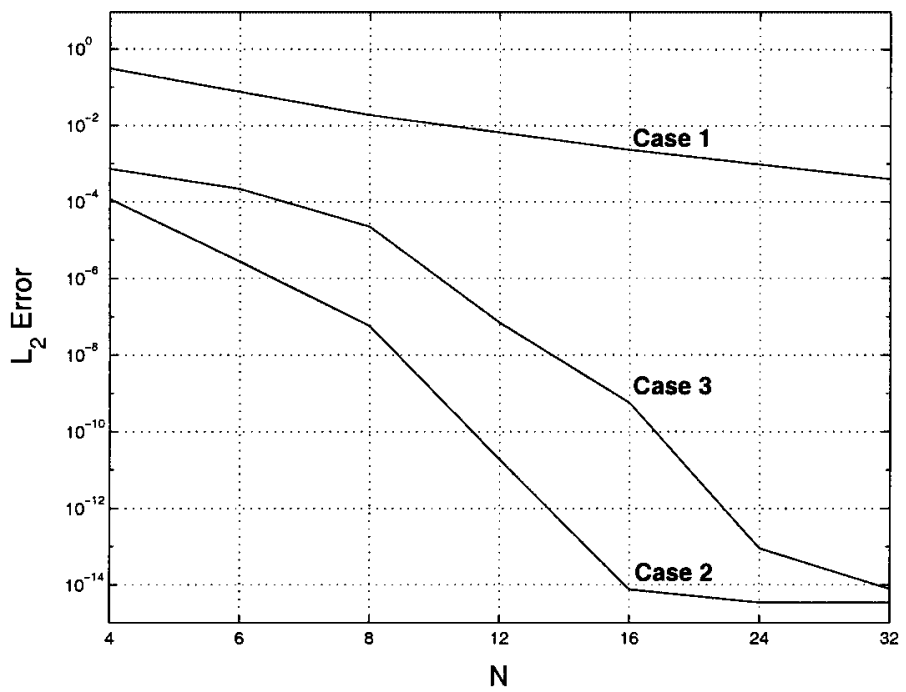

FIG. 6. The $\varphi$ error as a function of the spatial approximation order, $N$, for Cases 1, 2, and 3 after 12, 5, and 5 days of integrations, respectively. The $n_{I}=1$ grid is used. 
cases except for the NCAR spectral transform model. Our nodal DGM model compares to the NCAR spectral transform model as follows: it is equal in accuracy for Case $1\left(\mathcal{O}\left(10^{-2}\right)\right)$, more accurate for Case $2\left(\mathcal{O}\left(10^{-11}\right)\right.$ versus $\left.\mathcal{O}\left(10^{-9}\right)\right)$, and less accurate for Case $3\left(\mathcal{O}\left(10^{-8}\right)\right.$ versus $\left.\mathcal{O}\left(10^{-9}\right)\right)$. This comparison is based on an equivalent number of grid points to the NCAR T42 model given by our icosahedral grid $n_{I}=1, N=12\left(N_{\mathrm{p}}=8000\right)$.

However, in [18] the accuracy of the NCAR spectral transform model is shown to vary substantially depending on the direction of the flow with respect to the coordinate axes. This is particularly striking for Case 1 when the wave goes through the poles. Note that the direction of the waves has no effect on the accuracy of our DGM model because the poles are not treated in any special way due to the Cartesian coordinate system. Furthermore, unlike the spherical Gaussian grid the icosahedral grid has no preferred directions. At increased levels of resolution we find that our DGM model becomes more accurate than the NCAR model. Comparing our $N=32$ result with their T106 (Fig. 4.6 in [18]) we find the accuracy of our model to be $\mathcal{O}\left(10^{-3}\right)$ compared to $\mathcal{O}\left(10^{-2}\right)$ for the NCAR model. In fact, our model at this resolution is more accurate than their $\mathrm{T} 170$.

\subsubsection{Qualitative Study}

In the previous section we tested cases that have analytic solutions against which to compare and judge the accuracy of our nodal DGM scheme. However, the more relevant Cases 4, 5, and 6, do not allow such simple analytic solutions. Instead, we evaluate the convergence characteristics of the scheme qualitatively by running the cases using different resolutions. In addition, we show results for Case 7, which contains steep solution gradients such as those that might arise due to weather fronts.

In Figs. 7-9 the left panels show the contours of the mass, the middle panels illustrate the zonal velocity component, and the right panels show the meridional velocity component. The zonal velocity component is the $u$ component in spherical coordinates which is associated with the longitude $\lambda$ and is positive when traveling west to east in a direction parallel to the equator. The meridional velocity component is the $v$ component in spherical coordinates which is associated with the latitude $\theta$ and is positive when traveling from the south pole to the north pole.

Figure 7 shows the results for Case 4 for a 10-day integration using $N=8,16$, and 32 , respectively. The contours are shown from a viewpoint corresponding to the north pole $(\lambda, \theta)=(0,90)$. These results confirm that the wave structures remain the same for all three values of $N$. However, the wave pattern clearly becomes better resolved when increasing $N$. This is most obvious near the north pole (center of the plots) for the velocity components. The contours are a bit jagged for $N=8$ but become smoother for $N=16$. Finally, for $N=32$ we see that the same patterns exist as in the $N=8$ and $N=16$ contours but the curves are much smoother throughout, indicating a converged result.

Figure 8 shows the results of Case 5 for a 10-day integration at different resolutions with the contours shown from the viewpoint $(\lambda, \theta)=(180,0)$, where the peak of the mountain resides at $(\lambda, \theta)=(180,30)$. The flow impinging on the mountain causes the resulting wave structures, which we see in the figure around the midlatitudes $(\theta= \pm 45)$. The contours for $N=8$ are again a little jagged — not just for the mass contours but for the velocity components as well. Increasing $N$ to 16 removes much of the jaggedness visible in the contours and increasing $N$ further to 32 smooths the contours completely, resulting in clearly visible cohesive wave structures. 
a)
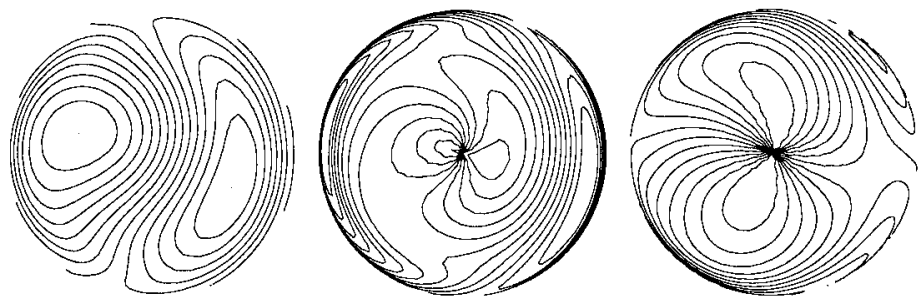

b)
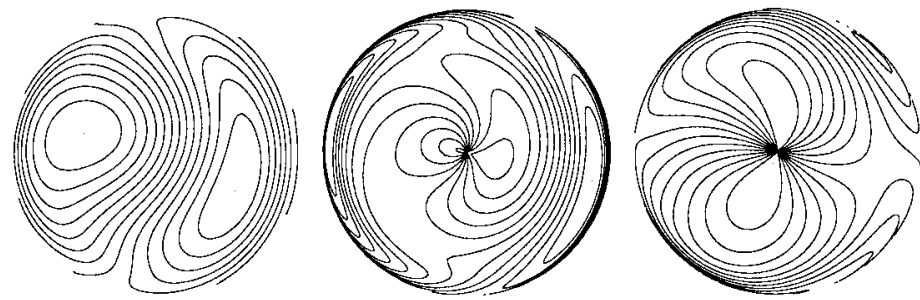

c)
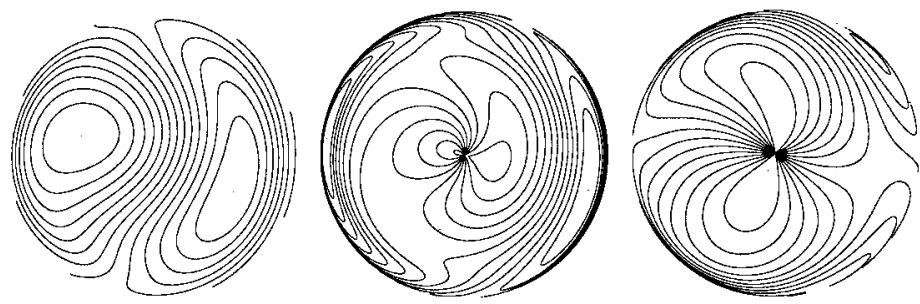

FIG. 7. Case 4. Contours of the mass (left), u-velocity (middle), and v-velocity (right) on grid $n_{I}=1$ and a) $N=8$, b) $N=16$, and c) $N=32$ after a 10-day integration viewed from $(\lambda, \theta)=(0,90)$.

Figure 9 shows the results of Case 6 after a 14-day integration at different resolutions with the contours shown from a viewpoint corresponding to the north pole $(\lambda, \theta)=(0,90)$. In contrast to the previous two cases, the results for $N=8$ show that the wave structures are beginning to break down at this resolution, which is insufficient to support the dynamics of the system. This breakdown of the wave structures is most obvious by looking at the mass. Increasing $N$ to 16 results in a dramatic improvement with the wave structures becoming more cohesive. This is particularly noticeable in the mass contours where we see that the rotated Greek cross pattern remains intact instead of breaking off into distinct blobs as in Fig. 9a. Increasing $N$ further to 32 significantly improves the wave patterns. The mass contours for the $N=16$ scheme are beginning to reveal a semblance of break-off of the wave pattern. This can be seen by looking at the bottom left tip of the cross. For $N=32$ the wave pattern remains completely intact without any indication of break-off.

The results for these three cases show that increasing the order of the approximation, $N$, either improves the smoothness of the contour curves or allows for the resolution of finer scale waves. This is particularly noticeable in Cases 5 and 6. In Case 5, the velocity components exhibit very localized wave formations that are extremely well resolved by our nodal $N$ th order DGM scheme. Case 6 illustrates the breakdown of the wave structure when insufficient grid resolution is used. 
a)
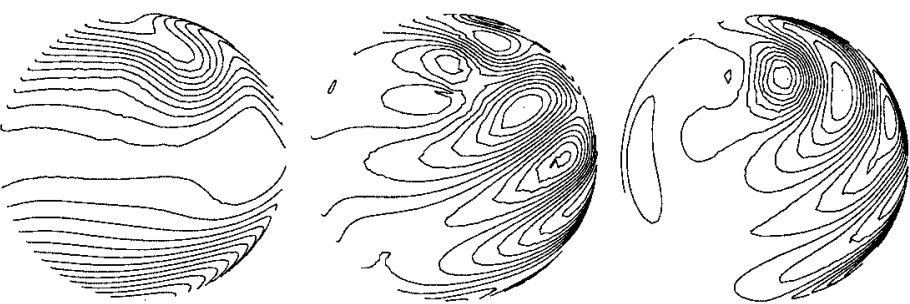

b)
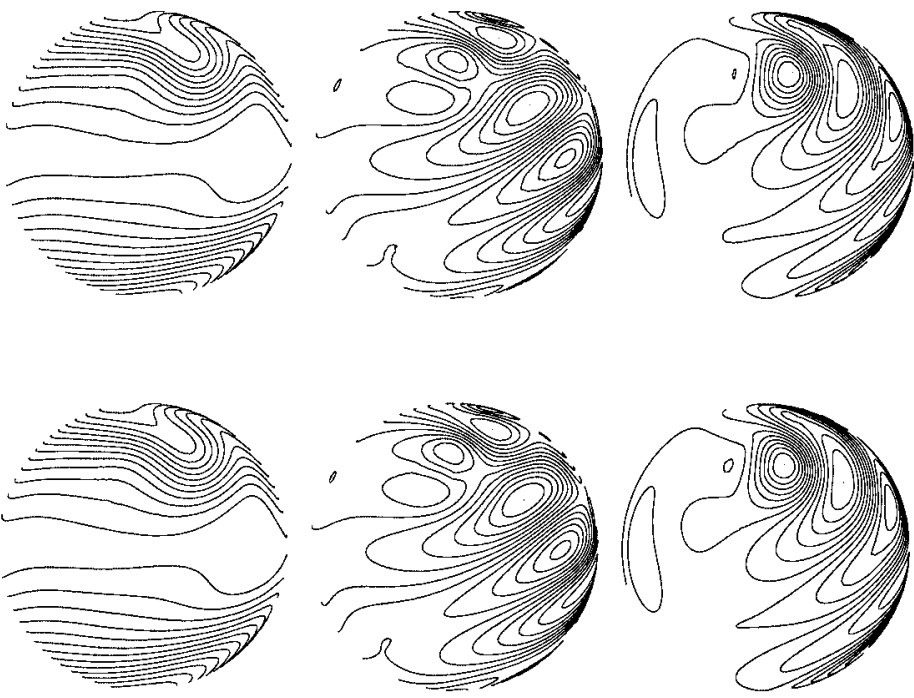

c)

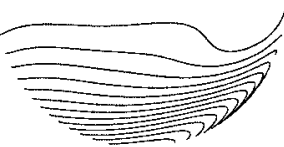

FIG. 8. Case 5. Contours of the mass (left), u-velocity (middle), and v-velocity (right) on grid $n_{I}=1$ and a) $N=8$, b) $N=16$, and c) $N=32$ after a 10-day integration viewed from $(\lambda, \theta)=(180,0)$.

Finally, we show results for a nonsmooth solution comparing the continuous spectral element method (SEM) and the discontinuous Galerkin method (DGM). Figures 10 and 11 show profiles of the mass along the Equator after one full revolution (12 days) using the same time step and grid $\left(n_{I}=1, N=16\right)$.

In Fig. 10 we compare the SEM and DGM formulations using no filtering. Note that the steep gradient produces Gibbs phenomena, which globally impacts the solution in the SEM formulation while the DGM formulation handles this gradient more gracefully by localizing the impact of the oscillations. However, in reality, we would not use the SEM form without filtering and so in Fig. 11 we compare the two methods with the Boyd-Vandeven filter applied every 10 time steps. The SEM formulation benefits greatly from filtering whereas it is unclear whether the DGM formulation benefits at all. For the very simple case of advection of a circular column, the filtered DGM seems to handle local gradients slightly more accurately than the filtered SEM. Part of the reason for the difference stems from the fact that the DGM form is locally conserving while the SEM formulation is only globally conserving. The property of local conservation may not be too important in global numerical weather prediction (except perhaps in hurricane tracking), but it will certainly be important in mesoscale models, which are used to simulate fine-scale flows involving fronts, density currents, and hydraulic jumps due to topography (e.g., mountains and coastlines; see [4]). 
a)
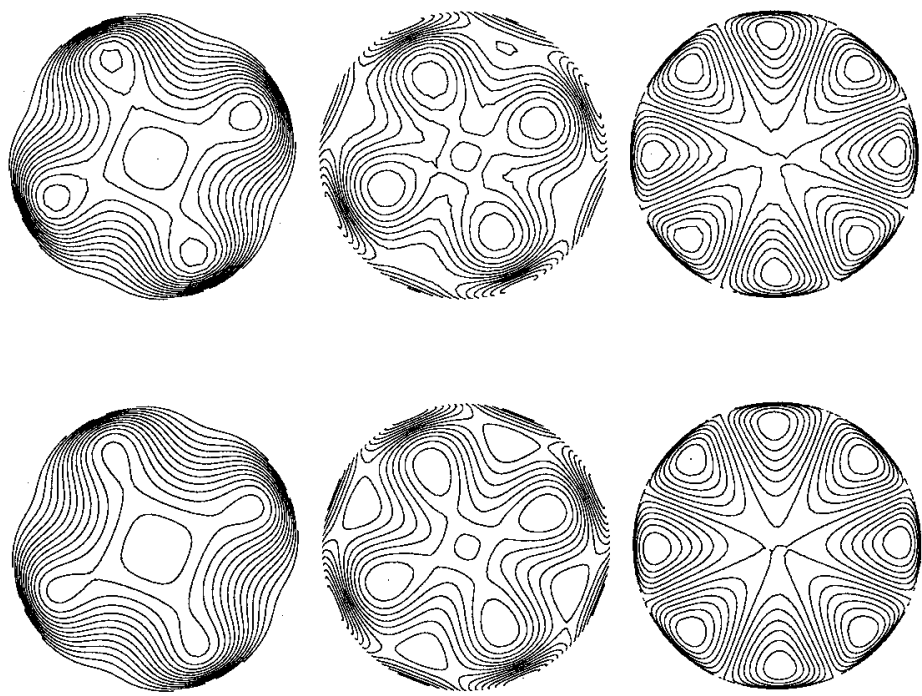

c)
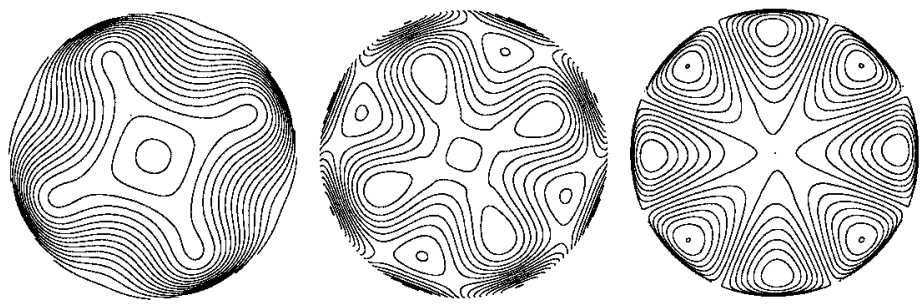

FIG. 9. Case 6. Contours of the mass (left), u-velocity (middle), and v-velocity (right) on grid $n_{I}=1$ and a) $N=8$, b) $N=16$, and c) $N=32$ after a 14-day integration viewed from $(\lambda, \theta)=(0,90)$.

\subsection{Impact of Filtering on Accuracy}

It is important to understand what effects, if any, filtering has on the accuracy of a particular scheme; this is especially important for high-order methods where filtering is often needed for solving nonlinear problems. In Fig. 12 we show the effects of filtering on

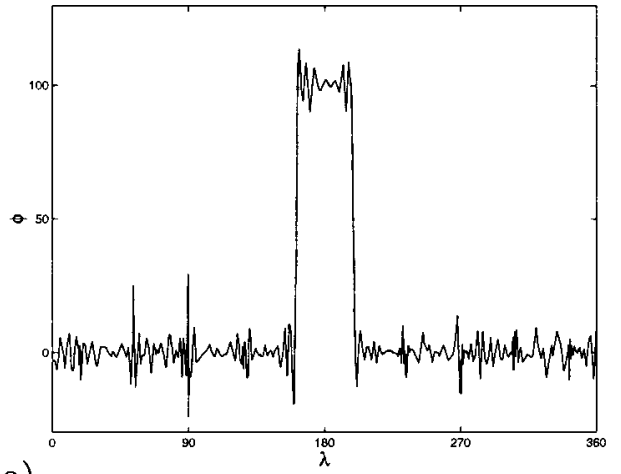

a)

FIG. 10. Case 7. The mass $\varphi$ along the equator after 12 days using $n_{I}=1$ and $N=16$ for a) SEM without filtering and b) DGM without filtering.

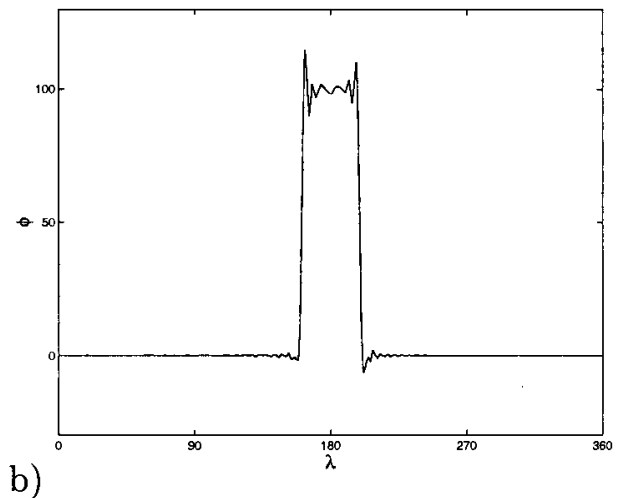

b) 


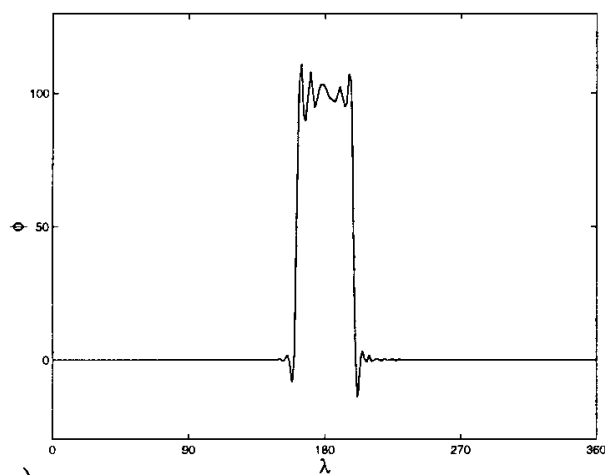

a)

FIG. 11. Case 7. The mass $\varphi$ along the equator after 12 days using $n_{I}=1$ and $N=16$ for a) SEM with filtering and b) DGM with filtering.

the convergence rate of Case 2. In this figure, we plot the solution after applying filtering every: 10 time steps (dashed), 100 time steps (dotted), 1000 time steps (dashed-dotted), and using no filtering (solid). We note that applying the filter too often (every 10 time steps) results in a decrease in accuracy of two orders of magnitude for the highest order scheme $(N=32)$. Clearly, we need to apply sufficient filtering to ensure stability without overdamping the solution. For all the test cases explored in this paper, we found the solution to be stable for the filtering time $T_{f} \leq 400 \Delta t$; however, for simplicity we apply the filter every 100 time steps for Cases 4,5 , and 6 . It should be mentioned that it is possible to avoid filtering altogether but this would require exact integration of the discrete operators in the DGM formulation. However, in practice it is far more economical to use inexact integration with filtering as is done in the continuous spectral element method.

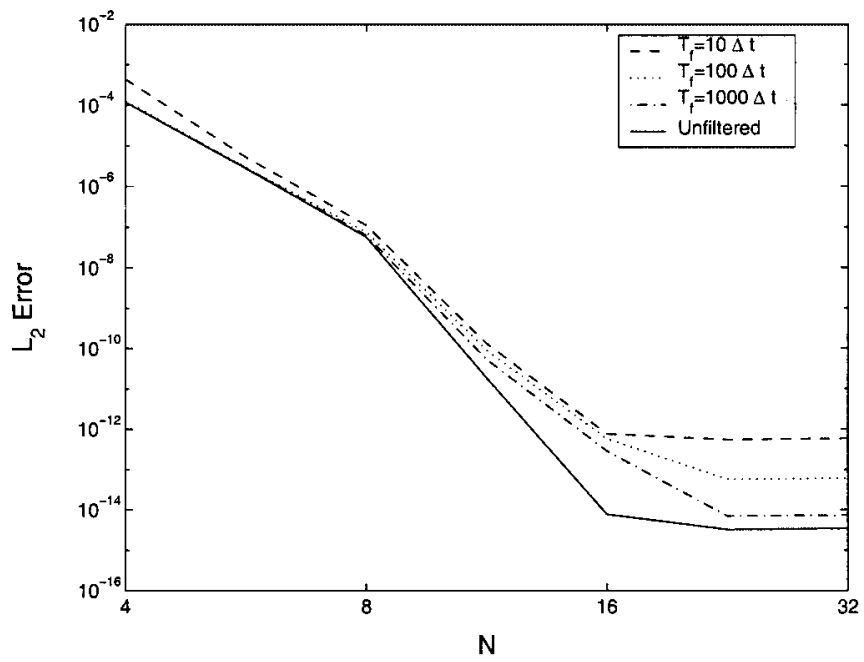

FIG. 12. The $\varphi$ error as a function of the spatial approximation order, $N$, with and without filtering. $T_{f}$ denotes the filter time: $T_{f}=10 \Delta t, 100 \Delta t$, and $1000 \Delta t$ represent filtering every 10 (dashed line), 100 (dotted line), and 1000 (dash-dotted line) time steps, respectively. Results are shown for Case 2 for a 5-day integration. 


\section{CONCLUSIONS}

The objective of this paper has been to present the formulation and verification of a highorder accurate nodal discontinuous Galerkin formulation for the solution of the spherical shallow water equations. Curvilinear quadrilateral elements are used to cover the sphere, using an icosahedral grid as the basis for the grid generation, with the equations solved in Cartesian form to avoid problems with coordinate singularities. On each curvilinear element, the solutions are represented by Lagrange polynomials in a purely nodal form, i.e., the solutions are given on quadrature points, and operations such as differentiation and integration become matrix-matrix operations. The equations are satisfied in a discontinuous element form with the element continuity being imposed weakly. This decouples all the elements and makes the formulation highly parallel as well as well-suited for adaptive solution techniques as no constraints on element conformity are needed.

The accuracy of the scheme, given in two mathematically equivalent but computationally different forms, has been illustrated by considering the solution of the standard set of benchmarks proposed in [32]. No significant difference was found between the performance of the two formulations although the divergence form seems more robust to performance enhancing approximations. The results confirm the expected high-order/spectral accuracy and illustrate the advantages of using such methods to efficiently solve geophysical flow problems.

To understand, however, whether the approach proposed here provides a realistic alternative to existing methods based on spherical harmonics or continuous spectral element methods requires extensive further validation. The inherent properties of the proposed technique, e.g., high parallel efficiency, high-order accuracy, and support for adaptive, nonconforming solution techniques, are sufficient to warrant such exhaustive studies and we hope to report on such results in the near future.

\section{ACKNOWLEDGMENTS}

The first author (FXG) gratefully acknowledges his sponsor, the Office of Naval Research, which supported this work through program element PE-0602435N. The two last authors acknowledge partial support of AFOSR/ DARPA under Contract F49620-1-0426. The second author (JSH) also acknowledges support by the Alfred P. Sloan Foundation as a Sloan Research Fellow and, furthermore, wishes to express his gratitude for the hospitality of the Department of Mathematical Modeling at the Technical University of Denmark in August 2001 during which parts of the present work were completed.

\section{REFERENCES}

1. F. Bassi and S. Rebay, High-order accurate discontinuous finite element solution of the 2D Euler equations, J. Comput. Phys. 138, 251 (1997).

2. K. S. Bey and J. T. Oden, hp-version discontinuous Galerkin methods for hyperbolic conservation laws, Computer Meth. Appl. Mech. Eng. 133, 259 (1996).

3. J. Boyd, The erfc-log filter and the asymptotics of the Euler and Vandeven sequence accelerations, Houston J. Math. (1996).

4. S. D. Burk and T. Haack, The dynamics of wave clouds upwind of coastal orography, Mon. Weather Rev. 128 , 1438 (2000).

5. B. Cockburn and C. W. Shu, The Runge-Kutta discontinuous Galerkin method for conservation laws: V. multidimensional systems, J. Comput. Phys. 141, 199 (1998).

6. B. Cockburn and C. W. Shu, Runge-Kutta discontinuous Galerkin methods for convection-dominated problems, J. Sci. Comput. 16, 145 (2001). 
7. B. Engquist and S. Osher, One-sided difference approximations for nonlinear conservation laws, Math. Comput. 36, 321 (1981).

8. F. X. Giraldo, Lagrange-Galerkin spectral element methods on unstructured quadrilateral grids, J. Comput. Phys. 147, 114 (1998).

9. F. X. Giraldo, Lagrange-Galerkin methods on spherical geodesic grids: the shallow water equations, J. Comput. Phys. 160, 336 (2000).

10. F. X. Giraldo, A spectral element shallow water model on spherical geodesic grids, Int. J. Numer. Meth. Fluids 35, 869 (2001).

11. F. X. Giraldo and T. E. Rosmond, A scalable spectral element Eulerian atmospheric model (SEE-AM) for NWP: Dynamical core tests, Mon. Weather Rev., in review.

12. D. Gottlieb and J. S. Hesthaven, Spectral methods for hyperbolic problems, J. Comput. Appl. Math. 128, 83 (2001).

13. J. J. Hack, B. A. Boville, B. P. Briegleb, J. T. Kiehl, P. J. Rasch, and D. L. Williamson, Description of the NCAR Community Climate Model (CCM2), NCAR Technical Note NCAR/TN-382 + STR (National Center for Atmospheric Research, Boulder, Calorado, 1992).

14. J. S. Hesthaven, A stable penalty method for the compressible Navier-Stokes equations. II. One-dimensional domain decomposition schemes, SIAM J. Sci. Comp. 18, 658 (1997).

15. J. S. Hesthaven, A stable penalty method for the compressible Navier-Stokes equations. III. Multi-dimensional domain decomposition schemes, SIAM J. Sci. Comp. 20, 62 (1999).

16. T. F. Hogan and T. E. Rosmond, The description of the Navy operational global atmospheric prediction systems spectral forecast model, Mon. Weather Rev. 119, 1786 (1991).

17. M. Iskandarani, D. B. Haidvogel, and J. P. Boyd, Staggered spectral element model with application to the oceanic shallow water equations, Int. J. Numer. Meth. Fluids 20, 393 (1995).

18. R. Jakob-Chien, J. J. Hack, and D. L. Williamson, Spectral transform solutions to the shallow water test set, J. Comput. Phys. 119, 164 (1995).

19. J. Levin, M. Iskandarani, and D. B. Haidvogel, A nonconforming spectral element ocean model, Int. J. Numer. Meth. Fluids 34, 495 (2000).

20. J. G. Liu and C. W. Shu, A high-order discontinuous Galerkin method for 2D incompressible flows, J. Comput. Phys. 160, 577 (2000).

21. H. Ma, A spectral element basin model for the shallow water equations, J. Comput. Phys. 109, 133 (1993).

22. A. McDonald and J. R. Bates, Semi-Lagrangian integration of a gridpoint shallow water model on the sphere, Mon. Weather Rev. 117, 130 (1989).

23. B. Neta, F. X. Giraldo, and I. M. Navon, Analysis of the Turkel-Zwas scheme for the two-dimensional shallow water equations in spherical coordinates, J. Comput. Phys. 133, 102 (1997).

24. J. Pedlosky, Geophysical Fluid Dynamics (Springer-Verlag, New York, 1987).

25. P. L. Roe, Approximate Riemann solvers, parameter vectors and difference schemes, J. Comput. Phys. 43, 357 (1981).

26. J. G. Sela, Spectral modeling at the National Meteorological Center, Mon. Weather Rev. 108, 1279 (1980).

27. A. J. Simmons, D. M. Burridge, M. Jarraud, C. Girard, and W. Wergen, The ECMWF medium-range prediction models development of the numerical formulations and the impact of increased resolution, Meteorol. Atmos. Phys. 40, 28 (1989).

28. M. Taylor, J. Tribbia, and M. Iskandarani, The spectral element method for the shallow water equations on the sphere, J. Comput. Phys. 130, 92 (1997).

29. H. M. Tufo and P. F. Fischer, Terascale Spectral Element Algorithms and Implementations, in Proceedings of the ACM/IEEE SC99 Conference on High Performance Networking and Computing (IEEE Computer Society, CDROM, 1999).

30. B. Van Leer, Towards the ultimate conservative difference scheme. V: A second order sequel to Godunov's method, J. Comput. Phys. 32, 101 (1979).

31. H. Vandeven, Family of spectral filters for discontinuous problems, J. Sci. Comput. 6, 159 (1991).

32. D. L. Williamson, J. B. Drake, J. J. Hack, R. Jakob, and P. N. Swarztrauber, A standard test set for numerical approximations to the shallow water equations in spherical geometry, J. Comput. Phys. 102, 211 (1992). 